\title{
The Relationship Between Fatigue and Lower Extremity Function in People with Multiple Sclerosis with the Absence of Clinical Disability
}

\author{
(1) Asiye Tuba Ozdogar1, (1) Seda Dastan1, (1) Ozge Ertekin², (1) Cavid Baba1, (1) Serkan Ozakbas3 \\ ${ }^{1}$ Graduate School of Health Sciences, Dokuz Eylul University, Izmir, Turkey \\ 2Dokuz Eylul University, School of Physical Therapy and Rehabilitation, Izmir, Turkey \\ ${ }^{3}$ Dokuz Eylul University Faculty of Medicine, Department of Neurology, Izmir, Turkey
}

\section{Abstract}

Objective: Impairment in the lower extremity function and mobility is a symptom often among people with multiple sclerosis (pwMS), even in the absence of clinical disability. Fatigue is one of the most common symptoms reported by at least $80 \%$ of pwMS during any disease. This study investigated the relationship between fatigue and lower extremity function, which is assessed by the Six Spot Step test (SSST) in pwMS with the absence of clinical disability.

Materials and Methods: A total of 477 pwMS with an Expanded Disability Status scale (EDSS) score of $\leq 1.5$ were included in the study. The SSST was used to evaluate the complex sensorimotor function of lower extremity function, such as lower extremity muscle strength, coordination, and balance. Participants with SSST performance above 8 seconds were classified as impaired. In addition, a Modified Fatigue Impact scale-5item version was used to evaluate perceived fatigue. Demographic (gender, age) and clinical data (disability level and disease duration) of the participants were obtained from interviews and medical records.

Results: Impairment in SSST performance was detected in 171 (35.85\%) pwMS. Univariate regression analysis revealed that fatigue was significantly associated with the SSST $(p<0.001)$. Furthermore, fatigue was still an associated factor in the multivariate regression analysis after adjusting for age, gender, and disability level $(p<0.001)$.

Conclusion: This study showed that even in the absence of disability, fatigue could be related to impairment in SSST performance. Considering the reflection of SSST performance on functional mobility, ambulation, and daily life in pwMS, it is essential to include the fatigue in the evaluation and treatment of pwMS with the absence of clinical disability.

Keywords: Lower extremity function, mobility, Six Spot Step test, fatigue, gender

\section{Introduction}

Multiple sclerosis (MS) is a chronic, progressive, demyelinating, and multifactorial disease of the central nervous system, causing various symptoms and signs (1). Impairment in the lower extremity function and mobility is a symptom quite often among pwMS. According to community-based studies, having walking difficulty is a most compelling symptom for both pwMS and their care partners (2). Martin et al. (3) investigated the balance and walking patterns of pwMS with no pyramidal impairment, according to the Expanded Disability Status scale
(EDSS) pyramidal functional system. As a result, even absence of clinical disability, pwMS have a slower, lower stride length, and more extended double limb support walking patterns than healthy controls. This finding was further confirmed by Liparoti et al. (4) by comparing the walking patterns of pwMS and healthy controls using a temporal-spatial gait analysis system. They showed that pwMS had changes in gait patterns in walking speed, step features such as length and width, and double support time. Besides, gait abnormalities and balance problems are occurring in advanced stages of MS and affect the pwMS with the absence of clinical disability (5).

Address for Correspondence: Asiye Tuba Ozdogar, Dokuz Eylul University, Graduate School of Health Sciences, Izmir, Turkey

Phone: +90 2324124939 E-mail: asiye.tuba.ozdogar@gmail.com ORCID-ID: orcid.org/0000-0003-0043-9374

Received: 25.06.2021 Accepted: 28.07.2021

'Copyright 2021 by the Journal of Multiple Sclerosis Research published by Galenos Publishing House. 
Fatigue is one of the most common symptoms reported by at least $80 \%$ of people with MS (pwMS) during any period of the disease regardless of disability level and duration (6). Even though the pathophysiological mechanism of fatigue is complicated and is still not completely understood, it is wellknown that fatigue is related to anxiety, depression, and sleep quality in pwMS (7). In addition, fatigue has adverse effects on physical performance and psychological state, and socioeconomic statuses such as early retirement, reduction in working hours, and use of health services (8). However, there is limited evidence and controversial results regarding the relationship between fatigue and lower extremity function in the MS population (9-13). Moreover, no study investigated this relationship in the pwMS with the absence of clinical disability.

Walking difficulties in pwMS could be related to many factors such as muscle weakness, cerebellar ataxia, dynamic balance impairment, and loss of coordination (14). The tests used in the clinical measurement, such as Timed 25 Foot Walk test, Timed and Up Go, and Six Minute Walk test, focus on walking speed, balance, and walking endurance (15). However, the Six Spot Step test (SSST), which was developed as the lower extremity equivalent of the 9-hole peg test, is a more complex test than unidirectional walking on a flat surface, which evaluates complex sensorimotor functions of walking such as lower extremity muscle strength, spasticity, coordination, and balance (16). For this reason, SSST could be better to detect the disability that may occur for any reason in lower extremity function than another clinical measurement. Therefore, the main aim of the current study was to investigate the relationship between fatigue and lower extremity function, assessed by SSST in pwMS with the absence of clinical disability. The second aim was to determine the other possible risk factors that could be associated with lower extremity function, such as the demographic and clinical characteristics of participants.

\section{Materials and Methods}

\section{Study Design}

This cross-sectional study was performed at the MS Clinic of Dokuz Eylul University. This study was performed in line with the principles of the Declaration of Helsinki (as revised in Brazil 2013). The data included in the analyses were collected from another open-ended study labeled, "Follow-up of physical, psychosocial and cognitive influences in persons with multiple sclerosis: a prospective cohort study" (ClinicalTrials.gov Identifier: NCT03878836). The study protocol was approved by the Non-invasive Research Ethics Board of Dokuz Eylul University Ethics Committee (protocol number: 2959-GOA and approval number: 2016/27-08). Furthermore, this study was accomplished in line with the principles of the Declaration of Helsinki (as revised in Brazil 2013), and written consent was received from all participants.

\section{Participants}

The inclusion criteria had MS based on the 2017 version of McDonald's criteria, $\leq 1.5$ scores on the EDSS (equal to no disability), and a relapse-free period of 30 days. The exclusion criteria were as follows; having another neurological disorder and having any orthopedic surgery history comprising the ankle-foot, knee, hip, or spine, affecting gait and balance.

\section{Outcome Measures}

Demographic and clinical characteristics of the pwMS, such as age, gender, and disease duration, were recorded from the medical report.

EDSS scoring, as the most common measure for the disability level in pwMS, was performed according to the neurological examination of functional systems, which consisted of pyramidal, cerebellar, brainstem, sensory, bladder, and bowel, visual and cerebral (17). The ambulatory state of the patients was also recorded. The same senior MS neurologist examined all participants and also calculated their global EDSS scores.

The Modified Fatigue Impact scale-5 items version presents an evaluation of the perceived fatigue level. It consists of 5 items, and participants rate questions from 0 (never) to 4 (almost always) according to experiences during the past four weeks. The total score ranged from 0 to 20. The increase in the total scores indicates higher impacts of fatigue on daily life (18). The original version of the Fatigue Impact scale was found as reliable and valid for Turkish pwMS (19).

The SSST evaluates the complex sensorimotor function of gaits, such as lower extremity muscle strength, coordination, and balance. The test includes six items of $20-\mathrm{cm}$ width circles and a $1 \times 5 \mathrm{~m}$ test path. Five wooden cylinder circles consisting of 134 grams are located in the center of each circle, without one at the starting point. The participants were asked to pushes the cylinder blocks out of the area as fast as possible using the lateral and medial surfaces of the foot alternately. Before the test, the dominant foot of the participant was questioned by the physical therapist (16). Therefore, the test started with the dominant foot and was performed as four trials (medial and lateral of the dominant leg, medial and lateral of the nondominant leg, respectively)-the average time of four trials as recorded as the SSST score. Participants with SSST performance above eight seconds were classified as impaired (20).

\section{Statistical Analysis}

The Kolmogorov-Smirnov test and histograms were used to check the normal distribution of data. The multivariate analysis of covariance test was performed to test the differences between the groups for impaired in SSST and those nonimpaired. Covariates included age, gender, the EDSS score, and disease duration. Univariate and multivariate logistic regression 
were used to determine whether impairment in SSST and age, gender, the EDSS score, disease duration, and fatigue are related. Statistical significance was set at p\&lt;0.05. Data were analyzed using the IBM ${ }^{\circledR}$ SPSS ${ }^{\circledR}$ Statistics software (Version 25.0. Armonk, NY: IBM Corp.) (Table 1).

\section{Result}

Four hundred and seventy-seven pwMS were included in the data analysis. Disability in SSST performance was detected in 171 (35.85\%) pwMS. There was a significant difference in age, gender, the EDSS score, disease duration, and fatigue between people with impairment in SSST and non-impaired participants $(p<0.05)$ (Table 1).

Univariate regression analysis revealed that fatigue was one of the related factors with SSST $(\mathrm{OR}=1.090 ; 95 \% \mathrm{Cl}: 1.048-1.135$, $\mathrm{p}<0.001)$. In addition, there was a relationship between the impairment in SSST performance and fatigue after adjustment for age, gender, the EDSS score, and disease duration (OR= 1.095; 95\% Cl: 1.047-1.146, $\mathrm{p}<0.001)$. Table 2 presents the detailed information related factors with the impairment in SSST performance.

\section{Discussion}

The current study has indicated that fatigue, gender, and age are related to SSST performance in pwMS with an absence of clinical disability. Additionally, according to the cut-off score of eight-second recommended by Callesen et al. (20) impairment in SSST performance was detected in 35.85\% of our participants. The recent systematic review, which describes the gait patterns of pwMS, showed that gait abnormalities detected via threedimensional capture systems occur even minimally disability group of MS (21). Ayan et al. (22) also reported gait impairment in pwMS with the absence of clinical disability using clinical measurement. Remarkably, this study emphasized that even pwMS with an absence of clinical disability have impairment in the lower extremity function. Because the SSST includes complex sensorimotor functions of walking such as lower extremity muscle strength, spasticity, coordination, and balance, it is worth evaluating the related factors that can affect the performance of SSST.

The present study includes the first assessment that evaluated the relationship between fatigue and lower extremity function in pwMS with the absence of clinical disability. Larocca (2) investigated the MS symptom that most affected daily life, which they experienced at least two times during one week. They showed that fatigue was the symptom that pwMS reported and challenging most, with a rate of \%76. Besides, Koch et al. (23) reported that being fatigue in MS is not related to age, disease duration, gender, disability level, and disease course. In addition, Kalron (10) classified the pwMS as fatigued and non-fatigued to examine the association between fatigue

Table 1. Demographic and clinical characteristics of the participants

\begin{tabular}{|c|c|c|c|c|c|c|}
\hline & All $(n=477)$ & $\begin{array}{l}\text { Impaired } \\
(n=171)\end{array}$ & $\begin{array}{l}\text { Non-impaired } \\
(n=306)\end{array}$ & $\mathbf{F}$ & $\mathbf{p}$ & $\begin{array}{l}\text { Observed } \\
\text { power }\end{array}$ \\
\hline Age (years) & $33.96(9.74)$ & $37.74(10.14)$ & $31.75(8.45)$ & 44.456 & $<0.001$ & 1.000 \\
\hline \multicolumn{7}{|l|}{ Gender, n (\%) } \\
\hline Female & 355 & $150(87.7)$ & $205(67.0)$ & \multirow{2}{*}{25.609} & \multirow{2}{*}{$<0.001$} & \multirow{2}{*}{0.999} \\
\hline Male & 122 & $21(12.3)$ & $101(33.0)$ & & & \\
\hline Disease duration (years) & $5.86(5.90)$ & $7.21(6.46)$ & $5.14(5.47)$ & 13.647 & $<0.001$ & 0.958 \\
\hline MFIS-5, possible range: 0-20 & $5.70(4.79)$ & $6.95(5.33)$ & $4.96(4.31)$ & 18.086 & $<0.001$ & 0.989 \\
\hline
\end{tabular}

Significant $\mathrm{p}$ values are presented in bold, values are presented as mean (SD) unless specified, EDSS: Expanded disability status scale, MFIS: Modified fatigue impact scale- 5-item version

\section{Table 2. Possible factors related to the impairment in SSST}

\begin{tabular}{|c|c|c|c|c|c|c|}
\hline & \multicolumn{6}{|c|}{ Impairment in Six Spot Step test } \\
\hline & OR & $95.0 \% \mathrm{Cl}$ & $\mathrm{p}$ & OR & $95.0 \% \mathrm{Cl}$ & $\mathrm{p}$ \\
\hline EDSS & 1.410 & $1.056-1.884$ & 0.020 & 1.014 & $0.729-1.411$ & 0.935 \\
\hline Disease Duration & 1.060 & $1.026-1.094$ & $<0.001$ & 1.025 & $0.987-1.065$ & 0.195 \\
\hline Fatigue & 1.090 & $1.048-1.135$ & $<0.001$ & 1.095 & $1.047-1.146$ & $<0.001$ \\
\hline
\end{tabular}

OR: Odds ratio, Cl: Confidence interval, SSST: Six spot step test 
and spatio-temporal parameters of walking by the treadmill. He showed that pwMS with fatigue had a smaller step, shorter stride length, prolonged stance, double support phase, and a shorter single support phase than pwMS without fatigue. As a result, they showed increased fatigue levels in the lower walking speed group than the normal walking speed group. Also, they reported a significant moderate negative correlation between perceived fatigue and walking speed during the short walking test, which is assessed with the 10-meter walking test. Similarly, we found that perceived fatigue is one of the related factors with the lower extremity function.

There is a difference between females and males regarding walking speed, cadence, and step length in the general population. Also, men have more hip and knee flexion range than women (24). Existing evidence shows that the female/ male ratio suffering MS is 2.3-3.5:1, and the progression is worse for men than women regardless of disease course (12). However, there are limited studies that investigate the effects of gender on the walking pattern in MS. Pau et al. (25) investigated the differences between men and women regarding spatiotemporal and kinematic gait parameters. They found that men have reduced ankle plantar-flexion, increased knee flexion, and hip flexion than women.

On the other hand, Klineova et al. (26) demonstrated no association between the Timed 25 Foot Walk test and gender. Interestingly, in our study, women have a longer SSST average time than men. This can be explained by men's hip and knee range of motion demonstrated by Pau et al. (25). While performing the SSST, during pushes the cylinder blocks out of the area, it could take advantage for the benefit of the men. It is desirable for future work, effects of the gender on lower extremity function in MS.

\section{Study Limitations}

There are some limitations to the current study. First, we did not use the kinetic and kinematic methods, giving more detailed information about impairment in the lower extremity function. Second, we did not assess the psychosocial aspect of the participants, such as depression, anxiety, or quality of life, affecting the perceived fatigue level. However, the strength of this study is that we used large sample sizes, which can give more solid results to the relationship between fatigue and lower extremity function.

\section{Conclusion}

This study includes the evidence that the walking performance of MS patients without a significant disability may decrease due to fatigue and showed that the SSST could be used to measure fatigue-related lower extremity performance reduction. Considering the reflection of SSST performance on functional mobility, ambulation, and daily life in pwMS, it is important to include the fatigue in the evaluation and treatment of pwMS with the absence of clinical disability.

Acknowledgments: The authors acknowledge the Multiple Sclerosis Research Association for assistance during the recruitment of the study.

\section{Ethics}

Ethics Committee Approval: The research protocol was approved by Dokuz Eylul University Ethics Committee (protocol number: 2959-GOA and approval number: 2016/27-08). This study was performed in line with the principles of the Declaration of Helsinki (as revised in Brazil 2013).

Informed Consent: Written consent was received from all participants.

\section{Authorship Contributions}

Surgical and Medical Practices: C.B., S.O., Concept: A.T.O., S.D., O.E., C.B., S.O., Design: A.T.O., S.D., O.E., C.B., S.O., Data Collection or Processing: A.T.O., S.D., Analysis or Interpretation: A.T.O., Literature Search: A.T.O., S.D., O.E., C.B., S.O., Writing: A.T.O., O.E., S.O.

Conflict of Interest: The authors declare that they have no known competing financial interests or personal relationships that could have appeared to influence the work reported in this paper.

Financial Disclosure: The authors declare that they have no known competing financial interests or personal relationships that could have appeared to influence the work reported in this paper.

\section{References}

1. Hunter SF. Overview and diagnosis of multiple sclerosis. Am J Manag Care 2016;22(Suppl 6):s141-s150.

2. Larocca NG. Impact of walking impairment in multiple sclerosis: perspectives of patients and care partners. Patient 2011;4:189-201.

3. Martin CL, Phillips BA, Kilpatrick TJ, Butzkueven H, Tubridy N, McDonald E, Galea MP. Gait and balance impairment in early multiple sclerosis in the absence of clinical disability. Mult Scler 2006;12:620-8.

4. Liparoti M, Della Corte M, Rucco R, Sorrentino P, Sparaco M, Capuano R, Minino R, Lavorgna L, Agosti V, Sorrentino G, Bonavita S. Gait abnormalities in minimally disabled people with Multiple Sclerosis: A 3D-motion analysis study. Mult Scler Relat Disord 2019;29:100-107.

5. Cameron MH, Nilsagard Y. Balance, gait, and falls in multiple sclerosis. Handb Clin Neurol 2018;159:237-250.

6. Minden SL, Frankel D, Hadden L, Perloffp J, Srinath KP, Hoaglin DC. The Sonya Slifka Longitudinal Multiple Sclerosis Study: methods and sample characteristics. Mult Scler 2006;12:24-38

7. Béthoux F. Fatigue and multiple sclerosis. Ann Readapt Med Phys 2006;49:265-271, 355-60. (English, French)

8. Krupp LB, Serafin DJ, Christodoulou C. Multiple sclerosis-associated fatigue. Expert Rev Neurother 2010;10:1437-1447.

9. Nogueira LA, Dos Santos LT, Sabino PG, Alvarenga RM, Santos Thuler LC. Factors for lower walking speed in persons with multiple sclerosis. Mult Scler Int 2013;2013:875648. doi: 10.1155/2013/875648. 
10. Kalron A. Association between perceived fatigue and gait parameters measured by an instrumented treadmill in people with multiple sclerosis: a cross-sectional study. J Neuroeng Rehabil 2015;12:34.

11. Motl RW, Sandroff BM, Suh Y, Sosnoff JJ. Energy cost of walking and its association with gait parameters, daily activity, and fatigue in persons with mild multiple sclerosis. Neurorehabil Neural Repair 2012;26:1015-1021.

12. Morris ME, Cantwell C, Vowels L, Dodd K. Changes in gait and fatigue from morning to afternoon in people with multiple sclerosis. J Neurol Neurosurg Psychiatry 2002;72:361-365.

13. Crenshaw SJ, Royer TD, Richards JG, Hudson DJ. Gait variability in people with multiple sclerosis. Mult Scler 2006;12:613-619.

14. Filli L, Sutter T, Easthope CS, Killeen T. Profiling walking dysfunction in multiple sclerosis: characterisation, classification and progression over time. Sci Rep 2018:8:4984

15. Bethoux F, Bennett S. Evaluating walking in patients with multiple sclerosis: which assessment tools are useful in clinical practice? Int J MS Care 2011:13:4-14.

16. Nieuwenhuis MM, Van Tongeren H, Sørensen PS, Ravnborg M. The six spot step test: a new measurement for walking ability in multiple sclerosis. Mult Scler 2006;12:495-500.

17. Kurtzke JF. Rating neurologic impairment in multiple sclerosis: an expanded disability status scale (EDSS). Neurology 1983;33:1444-14452.

18. D'Souza E. Modified Fatigue Impact Scale - 5-item version (MFIS-5). Occup Med (Lond) 2016:66:256-257

19. Armutlu K, Keser I, Korkmaz N, Akbiyik DI, Sümbüloğlu V, Güney Z, Karabudak R. Psychometric study of Turkish version of Fatigue Impact Scale in multiple sclerosis patients. J Neurol Sci 2007;255:64-68.
20. Callesen J, Cattaneo D, Brincks J, Kjeldgaard Jørgensen ML, Dalgas U. How do resistance training and balance and motor control training affect gait performance and fatigue impact in people with multiple sclerosis? A randomized controlled multi-center study. Mult Scler 2020;26:1420-1432.

21. Coca-Tapia M, Cuesta-Gómez A, Molina-Rueda F, Carratalá-Tejada M. Gait pattern in people with multiple sclerosis: a systematic review. Diagnostics (Basel) 2021;11:584

22. Ayan H, Altin Ertekin Ö, Kahraman T, Ozakbas S. Balance and gait impairment in persons with multiple sclerosis with the absence of clinical disability. Türk Nöroloji Dergisi 2020;26:224-229.

23. Koch M, Mostert J, Heerings M, Uyttenboogaart M, De Keyser J. Fatigue, depression and disability accumulation in multiple sclerosis: a crosssectional study. Eur J Neurol 2009;16:348-352.

24. Ko SU, Tolea MI, Hausdorff JM, Ferrucci L. Sex-specific differences in gait patterns of healthy older adults: results from the Baltimore Longitudinal Study of Aging. J Biomech 2011;44:1974-1979.

25. Pau M, Corona F, Pilloni G, Porta M, Coghe G, Cocco E. Do gait patterns differ in men and women with multiple sclerosis? Mult Scler Relat Disord 2017;18:202-208.

26. Klineova S, Farber R, Saiote C, Farrell C, Delman BN, Tanenbaum LN, Friedman J, Inglese M, Lublin FD, Krieger S. Relationship between timed 25foot walk and diffusion tensor imaging in multiple sclerosis. Mult Scler J Exp TransI Clin 2016;2:2055217316655365. doi: 10.1177/2055217316655365. 